KN Kartographische Nachrichten Journal of Cartography and Geographic Information 68. Jahrgang, Oktober 2018, Heft 5 Herausgeber: Deutsche Gesellschaft für Kartographie e.V. (DGfK) - Gesellschaft für Kartographie und Geomatik, ver durch den Präsidenten Prof. Dr. Manfred Weisensee

Organ der Deutschen Gesellschaft für Kartographie e.V. (DGFK), der Schweizerischen Gesellschaft für Kartografie (SGK) und der Osterreichischen Kartographischen Kommission (ÖKK) in der Osterreichischen Gesellschaft für Geographie (ÖGG).

Schriftleitung

Houptschriftleiter

Prof. Dr. rer. nat. habil. Mark Vetter (zuståndig für Aufsătze, Berichte). Hochschule für angewandte Wissenschaften Würzburg-Schweinfurt, Röntgenring 8, 97070 Würzburg: vetter@kartographische-nachrichten.de

Fon: 0049(0) $9313511-8249$

Weitere Schriftleiter

Prof. Dr. phil. Frank Dickmann (Zweiter Schriftleiter, zuständig für Nachrichten aus Hochschulen und Institutionen, Rezensionen). Ruhr-Universităt Bochum, Geographisches Institut, Postfach, 44780 Bochum; frank.dickmann@ @rub.de Fon: 0049(0) 234 32-23379; Fax: 0049(0) 234 32-14964 Dipl.-Ing. Andreas Gollenstede (zuständig für Geoinformation aktuell) Haareneschstr. 91, 26121 Oldenburg; kn@gollenstede.com Fon:0049(0) 4417779-545; Fax: 0049(0) 4417779-750 Dr.-Ing. Thomas Chudy (zuständig für DGfK-, SKG- und ÖGGNachrichten). Martin-Luther-Universitāt Halle-Wittenberg, Institut für Agrar- und Ernăhrungswissenschaften, Karl-Freiherr-vonFritsch-Straße 4, 06120 Halle/Saale, Fon: 0049(0) 3455522448 ; Fax: 0049(0) 3455527109

Prof. Dr. Reinhard Zölitz (zuständig für Nachrichten aus Hochschulen und Institutionen), Institut für Geographie und Geologie, Kartographie und GIS, Friedrich-Ludwig-Jahn-Str. 16,

D-17489 Greifswald, Fon 03834864523 , Fax: 03834864501 E-Mail: zoelitz@uni-greifswald.de

Mitarbeit für die Schweiz: Prof. Dr. Lorenz Hurni. Institut für Kartografie, ETH Hönggerberg, 8093 Zürich; hurni@karto.baug.ethz. ch. Fon: 0041 16333033; Fax: 00411631153

Mitarbeit für Österreich: Univ-Prof. Dr. Wolfgang Kainz

Institut für Geographie und Regionalforschung, Universität Wien, Universitätsstraße 7/5, 1010 Wien; wolfgang.kainz@univie.acat Fon: 0043 14277-8640; Fax: 0043 14277-9531

Ehrenschriftleiter

Prof. Dr. Jürgen Dodt t Witten

Dr. rer nat h. c. Rolf Harbeck Bonn

Editorial Board

Prof. Dr. H. Asche, Potsdam; Prof. Dr. Manfred Buchroithner, Dresden; Prof. Dr. Dirk Burghardt, Dresden; Prof. Dr. D. Dransch. Potsdam; Prof. Dr. M. Ehlers, Osnabrück, Berlin; Prof. Dr. S. I Fabrikant, Zürich, Schweiz; Ass.-Prof. Dr. S. Fuhrmann, Fairfax USA; Prof. Dr. G. Gartner, Wien, Österreich; Prof. Dr. D. Grünreich, Ronnenberg; Dr. Rolf Harbeck, Bonn; Prof. Dr. A. Hüttermann. Ludwigsburg: Prof. Dr. L Hurni, Zürich: Prof. Dr. W. Kainz. Wien; Ludwigsourg; Prof. Dr. L Humi, Zurich, Prof. Dr. W. Kainz, Wien; Prof. Dr.P. Kammere, Mänchen; Prof, Dr.W. G. Koch, Dresden Prof. Dr. Menno-Jan Kraak, Twente, Netherlands; Prof. Dr. Prof. Dr. J. Schiewe, Hamburg; PD Dr.-Ing. J. Schoppmeyer, Bonn: Prof. Dr. J. Schweikart, Berlin; Prof. Dr.-Ing. M. Sester, Hannover Prof. Dr. J. Siemer, Regina, Kanada; Prof. Dr. R. Zölitz, Greifswald. Manuskripte richten Sie bitte an den zuständigen Schriftleiter. Hinweise zur Gestaltung des Manuskripts finden Sie unter wwwkartographische-nachrichten de > Autorenhinweise. Reviewverfahren

Wissenschaftliche Beitrăge werden im Rahmen eines Peer-ReviewVerfahrens begutachtet. Trotz sorgfältiger Redigierung übernehmen Herausgeber, Redaktion und Verlag keine Haftung für die inhaltliche Richtigkeit der Veröffentlichungen.

Die KN Kartographische Nachrichten ist in der internationalen

Zitationsdatenbank Scopus gelistet.

Die DGfK ist Mitglied der

internationalen Kartographischen

Vereinigung IKV/ICA.

Verlag: Kirschbaum Verlag GmbH

Siegfriedstraße 28, D-53179 Bonn

Siegfriedstraße 28, D-53179 Bonn

Fon: (0228) 95453-0, Fax: (0228) 95453-27

Internet: $h$ ttp://www.kirschbaum.de, E-Mail: info@kirschbaum.de Anzeigenleiter: Michael Dietl (Nerlagsleiter Fachzeitschriften print Et digital), Fon: (0228) 95453-47, E-Mail: m.dietl@kirschbaum.de; Anzeigenpreisliste Nr. 53 vom 1.10.2017

Bezugspreise und ISSN: Inland/Ausland Jahresabonnement

inkl. E-Paper und elektronischem Archiv 74,- Euro zzgl.

Versand 9,80,- Euro (jeweils einschl. MwSt.).

Einzelheft 18,- Euro zzgl. Versand

Die Zeitschrift erscheint alle zwei Monate. ISSN 0022-9164. Kündigungsfrist 6 Wochen zum Auslaufen des AbonnementZeitraumes.

Satz: EMS Eckert Medienservice, 53881 Euskirchen

Druck: johnen-druck GmbH \& Co. KG, Industriegebiet Bornwiese, 54470 Bernkastel-Kues

\title{
Serious Gaming in der Geovisualisierung
}

\author{
Liebe Leserin, lieber Leser,
}

auch wenn ich in der ersten Ausgabe der $\mathrm{KN}$ in diesem Jahr schon im Vorwort auf die Potenziale der Virtual Reality für die Kartographie hingewiesen habe, möchte ich Ihnen nun ein ganz ähnliches Thema näherbringen: Serious Gaming. Gemeint ist das „ernsthafte Spielen“. Dies bezeichnet sozusagen die Verfolgung von ernstzunehmenden Zielen in der Anwendung oder Forschung mit spielerischem Charakter.

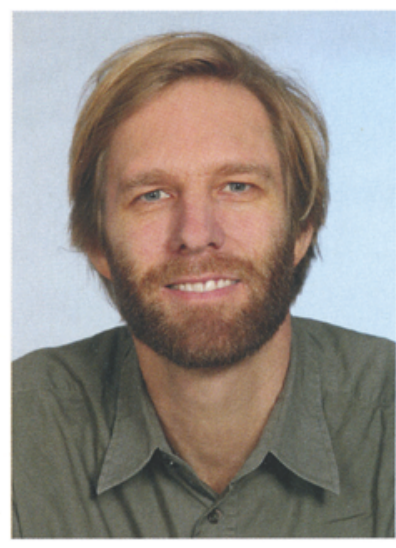

Mark Vetter

Die Entwicklung von Computerspielen läuft heutzutage häufig über Gaming Engines. Unity3D oder Unreal Engine wären in diesem Umfeld bekannte Programme. Mit diesen Gaming Engines „bauen“ sich die Entwickler virtuelle Realitäten (VR) im dreidimensionalen Raum. Dies können einzelne Räume sein, ganze Gebäudekomplexe, aber auch quasi-natürliche Landschaftsausschnitte. Das Besondere ist, dass es möglich ist, Figuren (Avatare) in diesen Umgebungen zu entwickeln, die aus der Perspektive der Nutzerin oder des Nutzers Aufgaben „spielerisch“ bewältigen müssen. Dieses Aufgabenspektrum reicht von einer Bewegung im virtuellen Raum, dem Aufsuchen bestimmter Objekte oder das Aufsammeln von Gegenständen bis zur Durchführung von bestimmten, virtuellen Handgriffen, um eine spezifische Aktion oder Prozess zu steuern. Idealerweise finden diese Aktivitäten von der Nutzerin oder dem Nutzer direkt mit einer VR-Brille, einem VR-Controller (Eingabegerät) und der entsprechend leistungsfähigen Computerhardware statt. Wichtig ist hier natürlich vor allem die schnelle Verarbeitung von Graphikdaten für das Display in der VR-Brille.

Dass diese Software zur Spieleentwicklung so eine schnelle Verbreitung in den letzten Jahren gefunden hat, liegt vor allem daran, dass die Programme frei verfügbar sind. Somit kann jede/r Spieleentwicklung betreiben. Probieren Sie es einfach mal aus! Im Internet gibt es zahlreiche Erklärvideos dazu. Man findet auch Workflows, wie man Geodaten in ein VR-Modell integriert.

Im ersten Beitrag des vorliegenden KN-Heftes wird unter der Autorenschaft von Dennis Edler das Potenzial der VR-Technologie für die sozialkonstruktivistische Landschaftsforschung dargestellt. Ein weiterer Beitrag von Volker Kaminske beschäftigt sich mit zeitbasierten Ansätzen aus der Geographie und deren Auswirkungen auf die Kartographie.

Viel Freude beim Lesen wünscht Ihnen Ihr

\section{Mucuster}

